\title{
Neural mechanisms of spatial working memory: Contributions of the dorsolateral prefrontal cortex and the thalamic mediodorsal nucleus
}

\author{
SHINTARO FUNAHASHI, KAZUYOSHI TAKEDA, and YUMIKO WATANABE \\ Kyoto University, Kyoto, Japan
}

\begin{abstract}
The dorsolateral prefrontal cortex (DLPFC) has been known to play an important role in working memory. Neurophysiological studies have revealed that delay period activity observed in the DLPFC is a neural correlate of the temporary storage mechanism for information and that this activity represents either retrospective or prospective information, although the majority represents retrospective information. However, the DLPFC is not the only brain area related to working memory. The analysis of neural activity in the thalamic mediodorsal (MD) nucleus reveals that the MD also participates in working memory. Although similar task-related activities were observed in the MD, the directional bias of these activities and the proportion of presaccadic activity are different between the MD and the DLPFC. These results indicate that, although the MD participates in working memory, the way it participates in this process is different between these two areas, in that the MD participates more in motor control aspects than the DLPFC does.
\end{abstract}

Working memory is a mechanism for short-term active storage of information, as well as for processing stored information (Baddeley, 1986). Working memory is a basic mechanism for many higher cognitive functions, including thinking, reasoning, decision making, and language comprehension. Therefore, understanding the neural mechanisms of working memory is crucial for understanding the neural mechanisms of these cognitive functions. As Goldman-Rakic (1987) initially proposed, the dorsolateral prefrontal cortex (DLPFC) has been known to play an important role in working memory. Neurophysiological studies in which nonhuman primates have been used have revealed that tonic sustained delay period activity is a neural correlate of the temporary storage mechanism for information (Funahashi \& Kubota, 1994; Fuster, 1997; Goldman-Rakic, 1987, 1996a, 1996b). Most delay period activity has been shown to have directional selectivity, so that delay period activity has been observed only when the visual cue has been presented at a particular location in the visual field (Funahashi, Bruce, \& Goldman-Rakic, 1989; Niki, 1974; Niki \& Watanabe, 1976; Rainer, Asaad, \& Miller, 1998; Rao, Rainer, \& Miller, 1997; Wilson, Ó Scalaidhe, \& Goldman-Rakic,

This study was supported by Grants-in-Aid for Scientific Research (12050222, 13210072, and 14380367) from the Japanese Ministry of Education, Science, Sports, Culture, and Technology to S.F. The 21st Century COE Program (D-2 to Kyoto University), MEXT, Japan also supported this study. K.T. is currently at the RIKEN Brain Science Institute, Saitama, Japan. Y.W. is currently at the National Institute of Advanced Industrial Science and Technology (AIST), Tsukuba, Japan. Correspondence concerning this work should be addressed to S. Funahashi, Department of Cognitive and Behavioral Sciences, Graduate School of Human and Environmental Studies, Kyoto University, Sakyo-ku, Kyoto 606-8501, Japan (e-mail: h50400@sakura.kudpc.kyoto-u.ac.jp).
1993). In addition, delay period activity has been observed only when a monkey performed correct responses. When the monkey made an error, delay period activity was not observed or was observed but truncated (Funahashi et al., 1989; Funahashi, Inoue, \& Kubota, 1997; Fuster, 1973; Niki \& Watanabe, 1976). In addition, delay period activity has been shown to represent either retrospective (e.g., sensory) or prospective (e.g., motor) information, although the majority of delay period activity represents retrospective information in the DLPFC (Funahashi, Chafee, \& Goldman-Rakic, 1993; Niki \& Watanabe, 1976; Takeda \& Funahashi, 2002). On the basis of these observations, delay period activity has been considered a neural correlate of the mechanism for temporary active storage of information in working memory (Funahashi \& Kubota, 1994; Fuster, 1997; Goldman-Rakic, 1987, 1996a, 1996b).

Although the DLPFC participates in working memory, the DLPFC is not the only brain structure related to working memory. Many other brain structures could also participate in working memory. For example, tonic sustained delay period activity similar to that observed in the DLPFC has been observed in the posterior parietal cortex (Chafee \& Goldman-Rakic, 1998; Gnadt \& Andersen, 1988), the inferior temporal cortex (Fuster, 1990; Miller, Lin, \& Desimone, 1993; Miyashita \& Chang, 1988), and the caudate nucleus (Hikosaka, Sakamoto, \& Usui, 1989) while monkeys performed working memory tasks (e.g., delayed response tasks, delayed matching-to-sample tasks, and memory-guided saccade tasks). Therefore, these areas could also participate in working memory.

In addition to these brain areas, the thalamic mediodorsal (MD) nucleus could also participate in working memory, because the MD is a major source of afferents 
to the prefrontal cortex, as well as a major target of efferents from the prefrontal cortex (Giguere \& GoldmanRakic, 1988; Goldman-Rakic \& Porrino, 1985; Kievit \& Kuypers, 1977; Rouiller, Tanne, Moret, \& Boussaoud, 1999). Furthermore, the MD is a key structure in the cortico-basal ganglia-thalamo-cortical loop that originates from and terminates in the prefrontal cortex (Alexander, DeLong, \& Strick, 1986). These results suggest that the MD plays an important role in cognitive processes. Several studies have indicated that the MD plays an important role in working memory. For example, recent human neuroimaging studies showed MD activation in delayed matching- or nonmatching-to-sample tasks (de $\mathrm{Zu}$ bicaray, McMahon, Wilson, \& Muthiah, 2001; Elliott \& Dolan, 1999) and the Wisconsin card-sorting test (Monchi, Petrides, Petre, Worsley, \& Dagher, 2001). Lesions to the MD in monkeys led to severe deficits in the delayed matching-to-sample task (Parker, Eacott, \& Gaffan, 1997) and the delayed response task (Isseroff, Rosvold, Galkin, \& Goldman-Rakic, 1982). Neurophysiological studies showed MD neurons exhibiting delay period activity while monkeys performed the delayed response task (Fuster \& Alexander, 1971, 1973). These results indicate that the MD is an important structure for understanding the neural mechanisms of working memory.

Recently, several neurophysiological studies of the MD in which oculomotor tasks have been used have been published (Sommer \& Wurtz, 2004; Tanibuchi \& GoldmanRakic, 2003). For example, Tanibuchi and Goldman-Rakic reported that MD neurons exhibited spatially selective task-related activity while monkeys performed an oculomotor delayed response (ODR) task. However, the characteristics of MD task-related activity have not yet been fully examined. For example, although we know that some MD neurons exhibit directional task-related activity, we do not know what information this directional task-related activity represents. These functional characteristics of taskrelated activity, especially delay period activity, have been fully analyzed using ODR tasks in the DLPFC (Funahashi, Bruce, \& Goldman-Rakic, 1989, 1990, 1991; Funahashi et al., 1993; Takeda \& Funahashi, 2002). In this study, we tried to compare neuronal activity quantitatively between the MD and the DLPFC and to determine the similarities and differences between the functional properties of these two areas. Therefore, we used the ODR task and its related tasks, which have been used for DLPFC studies, sampled neural activity homogeneously across the MD, and compared the characteristics of task-related activity between the DLPFC and the MD (Takeda \& Funahashi, 2002; Watanabe \& Funahashi, 2004a, 2004b).

\section{METHOD}

Four rhesus monkeys ( 2 for DLPFC studies and 2 for MD studies) were used. The experiments were approved by the Animal Research Committee at Kyoto University and were conducted in accordance with the Guide for the Care and Use of Laboratory Animals of the National Institutes of Health. The methods used in this experiment have been fully described in Takeda and Funahashi (2002) and Watanabe and Funahashi (2004a, 2004b).

\section{Behavioral Tasks}

The goal of the experiment was to compare the characteristics of task-related activity between the DLPFC and the MD. Therefore, we used the ODR task and the rotatory ODR (R-ODR) task to examine neural activity in both areas. During the experiment, the monkey sat quietly in a primate chair in a dark sound-attenuated room. The monkey faced a 21 -in. color TV monitor on which a fixation point and visual cues were presented. The monkey's eye positions were monitored with the magnetic search coil technique.

In the ODR task (Figure 1A, left), the monkey was required to make a memory-guided saccade to the location at which the visual cue had been presented. After a 5-sec intertrial interval, a fixation point (FP; a small white circle) was presented at the center of the TV monitor. If the monkey continued to look at the FP for $1 \mathrm{sec}$ (fixation period), a visual cue (a white circle) was presented for $0.5 \mathrm{sec}$ (cue period) at one of eight predetermined locations around the FP randomly (Figure 1B). The monkey was required to maintain fixation on the FP throughout the 0.5 -sec cue period and the subsequent 3-sec delay period. At the end of the delay period, the FP was extinguished. This was the go signal for the monkey to make a saccade within $0.4 \mathrm{sec}$ (response period) to the location at which the visual cue had been presented. If the monkey performed the correct eye movement, a drop of liquid reward was given.

In the R-ODR task (Figure 1A, right), the monkey was required to make a saccade $90^{\circ}$ clockwise from the direction in which a visual cue had been presented. After a 5-sec intertrial interval, the FP (a small white + ) was presented at the center of the TV monitor. If the monkey continued to look at the FP for $1 \mathrm{sec}$ (fixation period), a visual cue (a white circle) was presented for $0.5 \mathrm{sec}$ (cue period) at one of four predetermined locations around the FP randomly (Figure 1B). The monkey was required to maintain fixation at the FP throughout the 0.5 -sec cue period and the subsequent 3 -sec delay period. At the end of the delay period, the FP was extinguished. This was the go signal for the monkey to make a saccade within $0.4 \mathrm{sec}$ (response period) $90^{\circ}$ clockwise from the direction in which the visual cue had been presented. If the monkey made a correct saccade, a drop of liquid reward was given. The eccentricity of the cue location was $17^{\circ}$.

All the monkeys performed both tasks at a more than $80 \%$ correct ratio most of the time. For details regarding the monkeys' behavioral performances, see Takeda and Funahashi (2002) and Watanabe and Funahashi (2004a).

\section{Task-Related Activity}

To determine whether a neuron exhibited task-related activity, we first inspected rasters and histograms aligned at several task events for each cue condition in the ODR task and then conducted statistical analyses. To obtain the neuron's baseline discharge rate, we calculated the mean discharge rate during the last $500 \mathrm{msec}$ of the fixation period for each cue condition. For cue period activity, we calculated the mean discharge rate during the $300-\mathrm{msec}$ period (from 50 to $350 \mathrm{msec}$ after the onset of the visual cue) for each cue condition. If the mean discharge rate during the cue period differed significantly from the baseline discharge rate by the Mann-Whitney $U$ test $(p<.05)$, we considered that the neuron had exhibited cue period activity. For delay period activity, we calculated the mean discharge rate during the 3 -sec delay period for each cue condition. If the mean discharge rate differed significantly from the baseline discharge rate by the Mann-Whitney $U$ test $(p<.05)$, we considered that the neuron had exhibited delay period activity. For response period activity, we first searched for the trial condition in which the maximum response period activity was observed and determined the period during which the peak activity was observed, using histograms aligned at the initiation of the saccade by visual inspection. We then calculated the mean discharge rate during the $300-\mathrm{msec}$ response period $(150 \mathrm{msec}$ before and $150 \mathrm{msec}$ after the period during which the peak activity was observed) for each cue condition. If the mean discharge rate differed significantly from the 
A

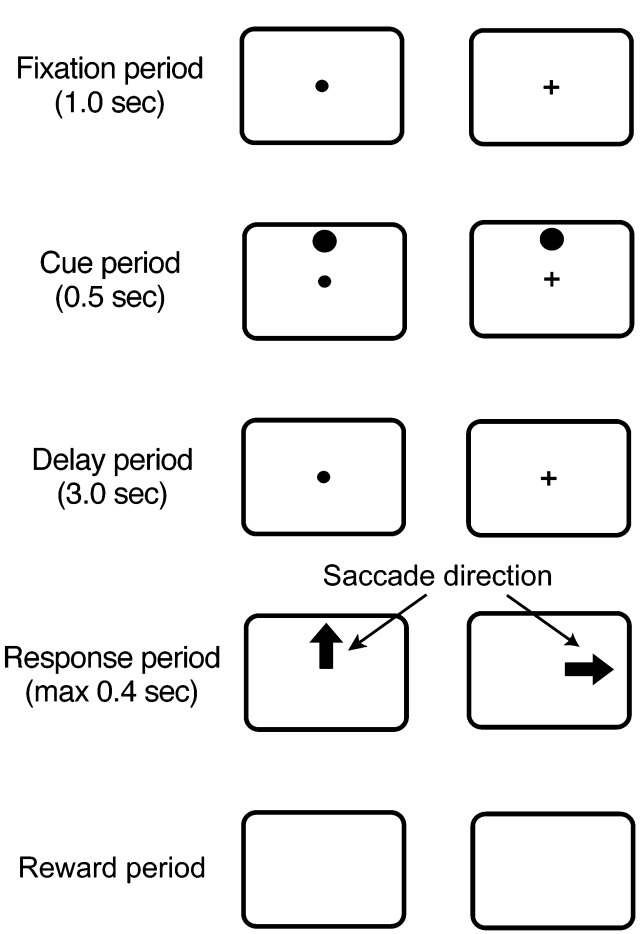

B ODR task

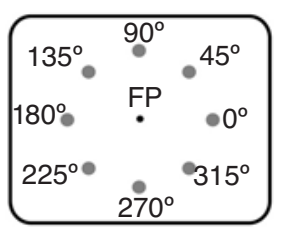

Figure 1. (A) Schematic drawings of an oculomotor delayed response (ODR) task and a rotatory ODR (R-ODR) task. (B) Positions of the visual cue used for these tasks. Eccentricity was $17^{\circ}$.

baseline discharge rate by the Mann-Whitney $U$ test $(p<.05)$, we determined that the neuron had exhibited response period activity. In addition, we also classified response period activity into two groups (pre- and postsaccadic activity) by examining neuronal activities aligned at the initiation of the saccade.

\section{NEURAL ACTIVITY DURING ODR PERFORMANCES}

\section{General Characteristics}

Three typical task-related activities (cue, delay, and response period activity; see Figure 2) have been observed in both the DLPFC and the MD while monkeys perform the ODR task. Cue period activity is the phasic excitatory response that occurred during the visual cue presentation. This activity has been shown to be a visual response observed in DLPFC neurons (Funahashi et al., 1990). Delay period activity is the tonic sustained activation observed during the delay period. This activity starts after the visual cue presentation, is maintained during the delay period, and terminates just after the initiation of the saccadic eye movement. Although the majority of neurons having delay period activity exhibit sustained activation during the delay period, some neurons exhibit a gradual increase or decrease of activation or tonic suppression during the delay period. These four types of delay period activity have been observed in both areas with similar proportions. Response period activity is the phasic excitatory response observed during the response period. This activity can be classified into two groups (presaccadic activity and postsaccadic activity) depending on whether the activity starts before or after the initiation of the saccadic eye movement, respectively.

Figure 3 shows Venn diagrams showing the proportions of task-related activity. Although all three types of taskrelated activity were observed in both the DLPFC and the $\mathrm{MD}$, the proportions of task-related activity were somewhat different between these two areas. In the DLPFC, among 160 task-related neurons, $38 \%, 47 \%$, and $61 \%$ exhibited cue, delay, and response period activity, respectively (Takeda \& Funahashi, 2002). Among these, 22 had only cue period activity, 33 had only delay period activity, and 45 had only response period activity. The remaining 60 (38\%) exhibited task-related activity during two or more task periods. Similarly, Funahashi et al. (1990) reported that, among 261 task-related DLPFC neurons, $28 \%, 64 \%$, and $56 \%$ exhibited cue, delay, and response period activity, respectively. Although 156 neurons $(60 \%)$ exhibited cue $(n=21)$, delay $(n=73)$, or response period $(n=62)$ activity, the remaining $105(40 \%)$ exhibited task-related activity during two or more task periods. On the other hand, in the MD (Watanabe \& Funahashi, 2004a), among 141 task-related neurons, 26\%, $53 \%$, and $84 \%$ exhibited cue, delay, and response period 


\section{DLPFC neurons}

Cue period activity

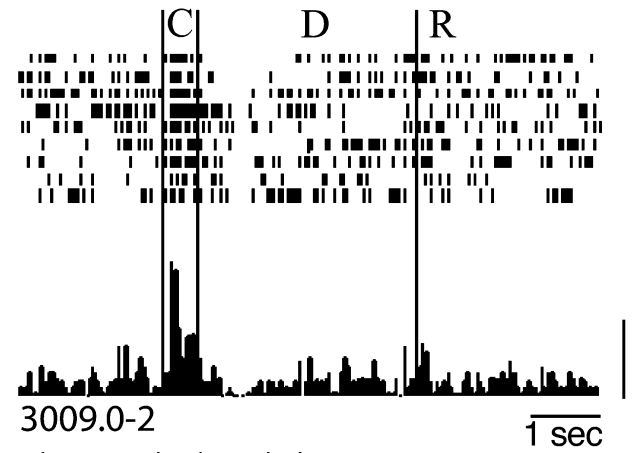

50 spikes/sec

Delay period activity

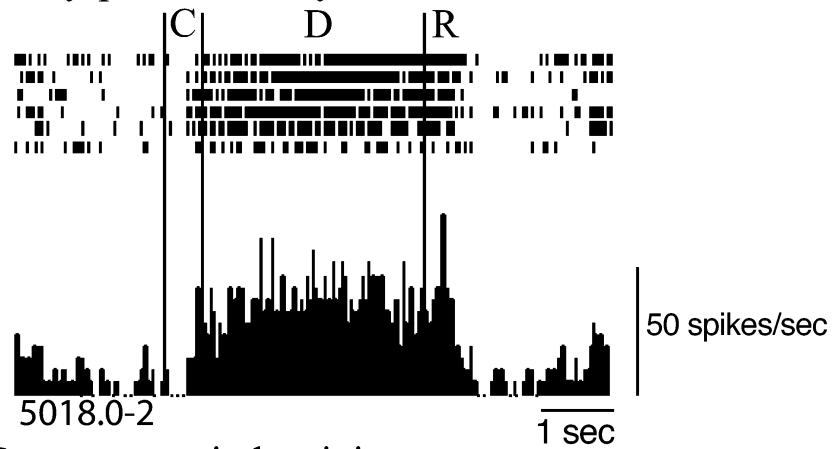

Response period activity

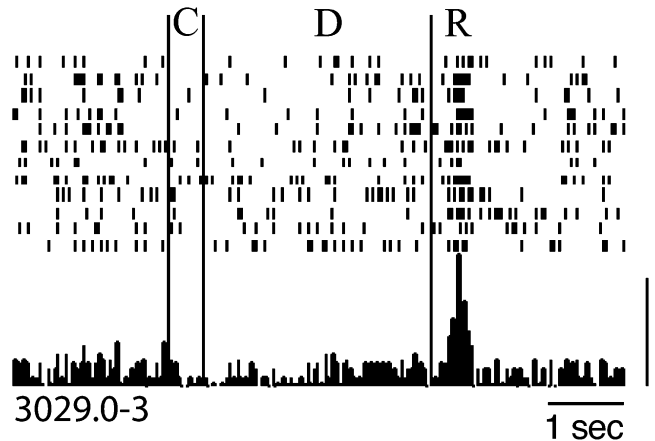

MD neurons

Cue period activity

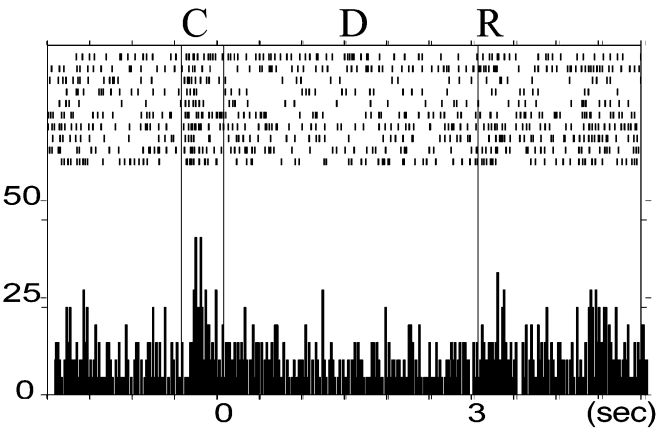

Delay period activity

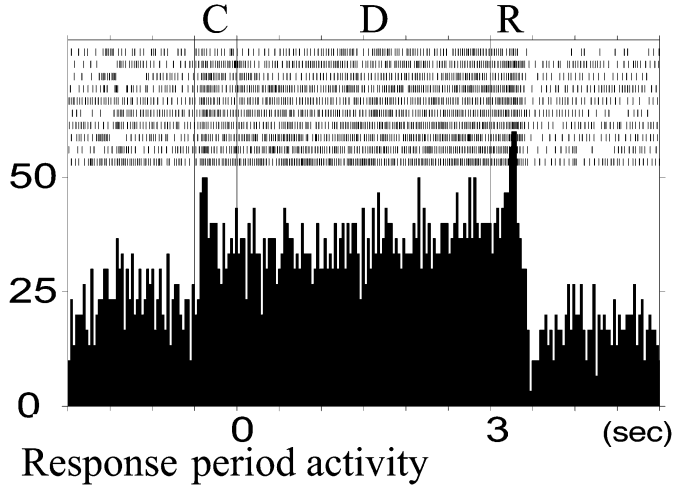

D R

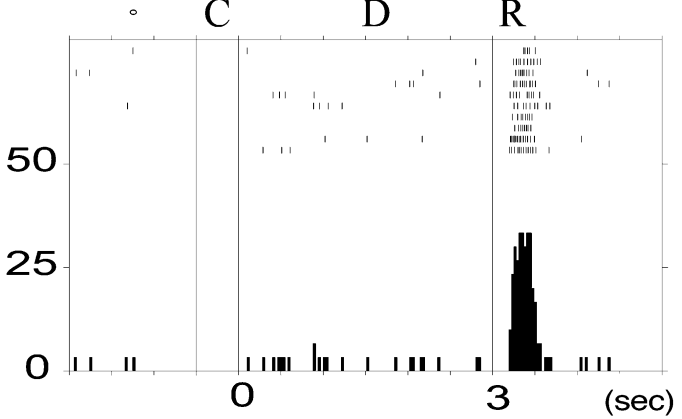

Figure 2. Three typical examples of task-related activity observed during oculomotor delayed response task performances. $C, D$, and $R$ indicate the cue period, the delay period, and the response period, respectively. The length of the delay period was 3 sec. DLPFC, dorsolateral prefrontal cortex; MD, mediodorsal nucleus.

activity, respectively. Among these, 5 had only cue period activity, 16 had only delay period activity, and 54 had only response period activity. The remaining 66 $(47 \%)$ exhibited task-related activity during two or more task periods.

These results indicate that although the proportion of neurons exhibiting delay period activity is similar between the DLPFC and the MD $\left(\chi^{2}=0.311, p>.05\right)$, more neurons exhibit cue period activity in the DLPFC, whereas more neurons exhibit response period activity in the $\operatorname{MD}\left(\chi^{2}=3.264, p<.05\right)$. In addition, most of the MD neurons having cue period activity exhibit response period activity (55\% in DLPFC neurons vs. $81 \%$ in MD neurons; $\left.\chi^{2}=4.970, p<.05\right)$. A similar tendency was observed in the MD neurons having delay period activity (49\% in DLPFC neurons vs. $76 \%$ in MD neurons; $\left.\chi^{2}=5.832, p<.02\right)$. These results suggest that, although the MD participates in working memory, the MD participates more in motor aspects than the DLPFC does.

\section{Directional Selectivity of Cue and Delay Period Activity}

It has been shown that most task-related activity exhibits directional selectivity. Directional selectivity can be defined by showing that neurons exhibited statistically significant task-related activity only when the vi- 

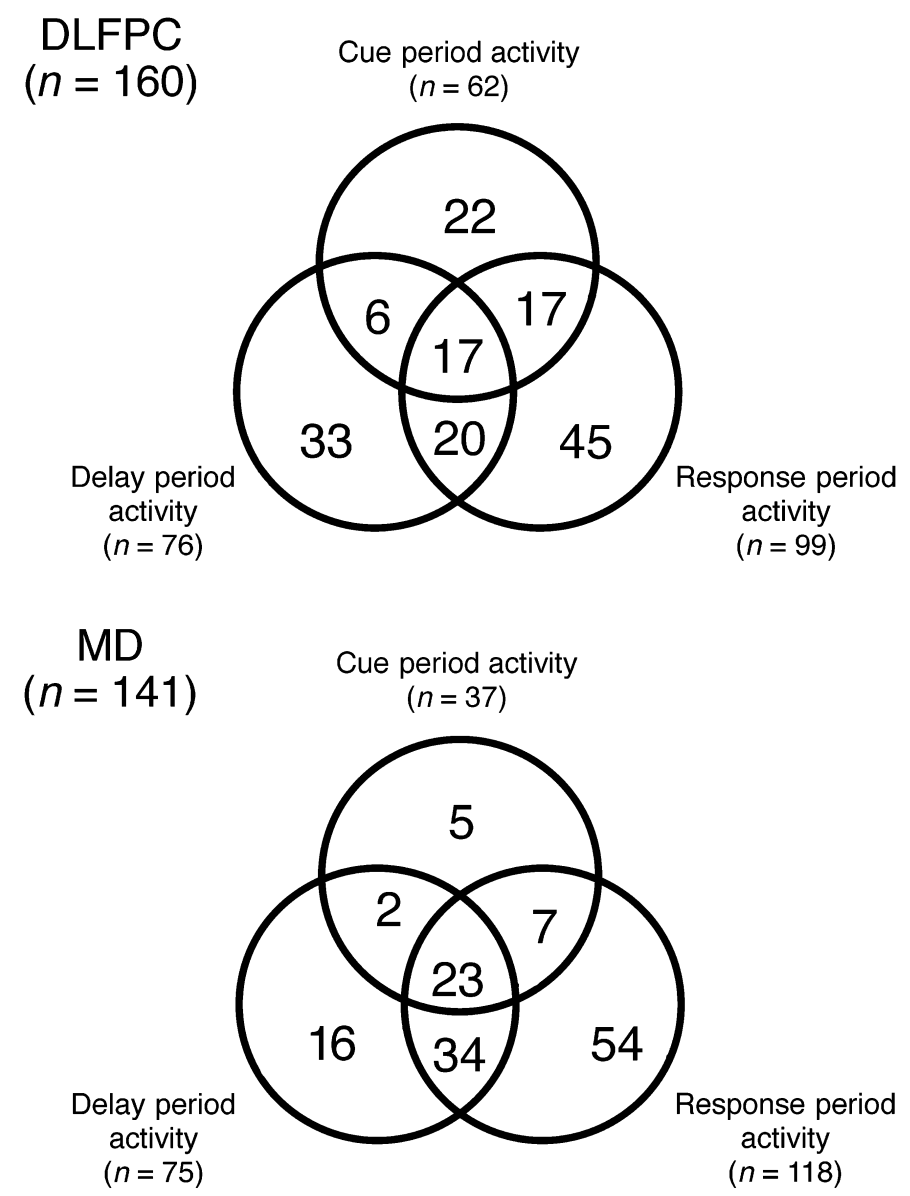

Figure 3. Venn diagrams showing the numbers of neurons exhibiting task-related activity. The dorsolateral prefrontal cortex (DLPFC) data are based on Takeda and Funahashi (2002). The mediodorsal (MD) nucleus data are based on Watanabe and Funahashi (2004a).

sual cues were presented in a particular area in the visual field or when monkeys made saccades toward particular directions. To quantify the directional selectivity of taskrelated activity, we constructed a tuning curve of this activity. The tuning curve was constructed from the mean discharge rate of task-related activity under each cue condition by its best fit to the Gaussian function. We determined the best direction for each directional taskrelated activity and compared the distribution of the best directions between the DLPFC and the MD.

As can be seen in Figure 4, most cue period activity has been shown to exhibit directional selectivity in DLPFC neurons (Funahashi et al., 1990; Takeda \& Funahashi, 2002), as well as in MD neurons (Watanabe \& Funahashi, 2004a). Best directions for cue period activity were mostly directed toward the contralateral visual field in both DLPFC neurons and MD neurons (Figure 5). The contralateral bias was statistically significant in both areas.

Similarly, most delay period activity exhibited directional selectivity (Figure 4). In the DLPFC, Takeda and Funahashi (2002) showed that $93 \%$ of delay period activity was directionally selective, whereas the remaining
7\% was omnidirectional. Similarly, Funahashi et al. (1989) showed that $79 \%$ was directionally selective, whereas $21 \%$ was omnidirectional. In the MD, $76 \%$ of delay period activity was directionally selective, whereas $24 \%$ was omnidirectional (Watanabe \& Funahashi, 2004a). The best directions for delay period activity were distributed in most of the directions around the FP in both DLPFC and MD neurons (Figure 5). In DLPFC neurons, the best directions were mostly toward the contralateral visual field, and the contralateral bias of the best directions was statistically significant $\left(\chi^{2}=8.758, p<.01\right.$; Funahashi et al., 1989). However, in MD neurons (Watanabe \& Funahashi, 2004a), 57\% had the best directions toward the visual field contralateral to the recording hemisphere, and 39\% had the best directions toward the visual field ipsilateral to the recording hemisphere (Figure 5). This contralateral bias was not significant $\left(\chi^{2}=\right.$ $3.375, p>.05)$.

\section{Characteristics of Response Period Activity}

Response period activity was observed in many neurons in both the DLPFC and the MD (Figure 3). How- 


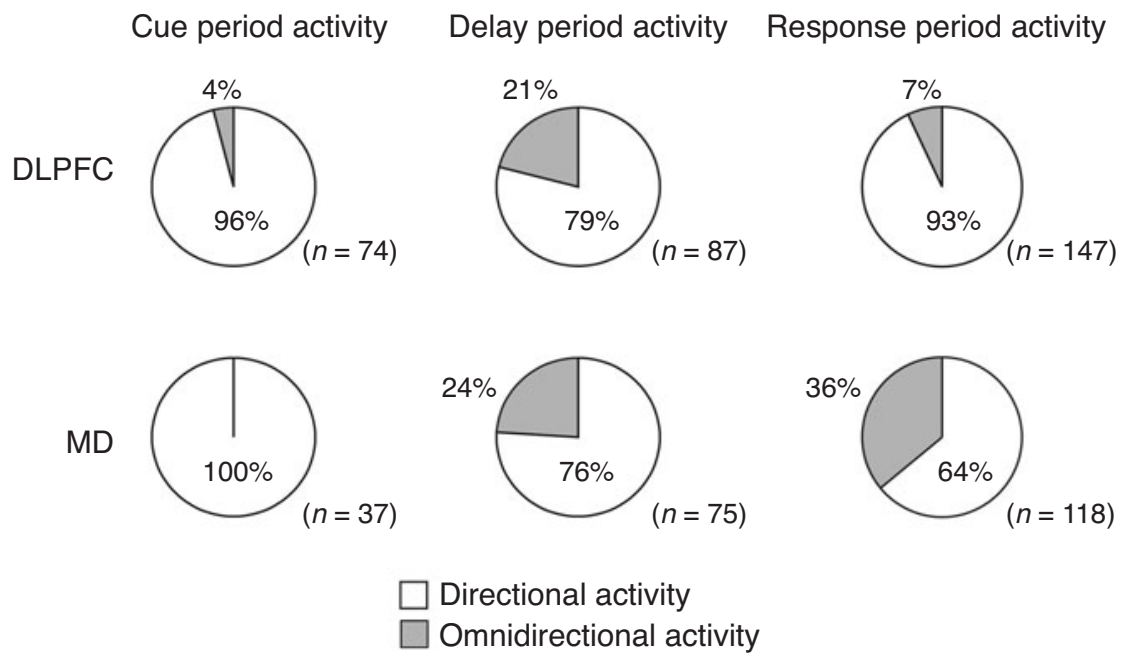

Figure 4. Directional selectivity of task-related activity for dorsolateral prefrontal cortex (DLPFC) and mediodorsal (MD) neurons. The DLPFC data are based on Takeda and Funahashi (2002). The MD data are based on Watanabe and Funahashi (2004a).

ever, the characteristics of response period activity were different between the DLPFC and the MD. Response period activity can be classified into two groups (pre- and postsaccadic activity) on the basis of whether this activity was initiated before or after the initiation of the saccadic eye movement, respectively. In the DLPFC, the latencies for response period activity were distributed from -190 to $340 \mathrm{msec}$; the mean was $63 \mathrm{msec}$ and the median was $70 \mathrm{msec}$ after the initiation of the saccadic eye movement (Takeda \& Funahashi, 2002). As can be seen in Figure 6, the great majority (78\% in Funahashi et al., 1991; 84\% in Takeda \& Funahashi, 2002) of DLPFC neurons exhibited postsaccadic activity. All DLPFC neu- rons having presaccadic activity exhibited directional selectivity (Figure 7). Among the DLPFC neurons having presaccadic activity, $61 \%$ had the best directions toward the visual field contralateral to the recording hemisphere, whereas $23 \%$ had the best directions toward the visual field ipsilateral to the recording hemisphere. The contralateral bias was statistically significant $\left(\chi^{2}=17.19\right.$, $p<.01)$. Most $(75 \%)$ of the DLPFC neurons having postsaccadic activity also exhibited directional selectivity (Figure 7). However, $48 \%$ of the neurons having postsaccadic activity had the best directions toward the visual field contralateral to the recording hemisphere, whereas $36 \%$ had the best directions toward the visual field ipsi-

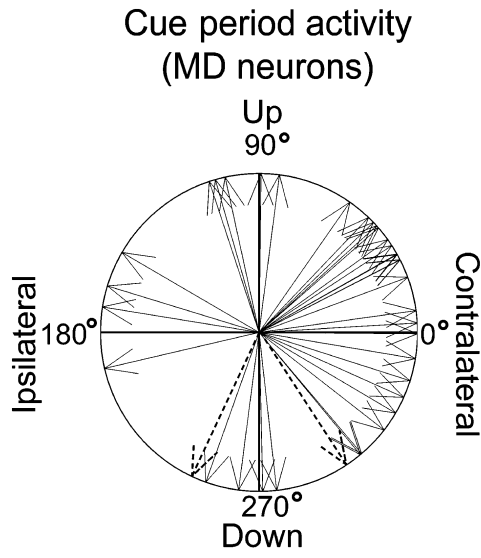

Excitatory activity
Inhibitory activity
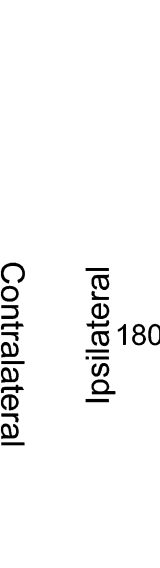
Excitatory activity
-.... Inhibitory activity

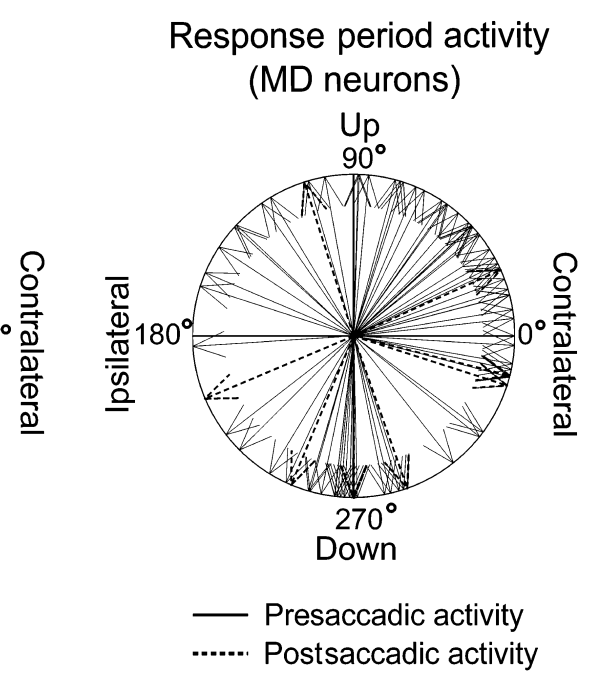

Figure 5. Polar distributions for the best directions for three task-related activities for mediodorsal (MD) neurons. The best directions of neurons recorded from the right thalamus were transformed into mirror image directions, as if all the neurons were recorded from the left thalamus. 

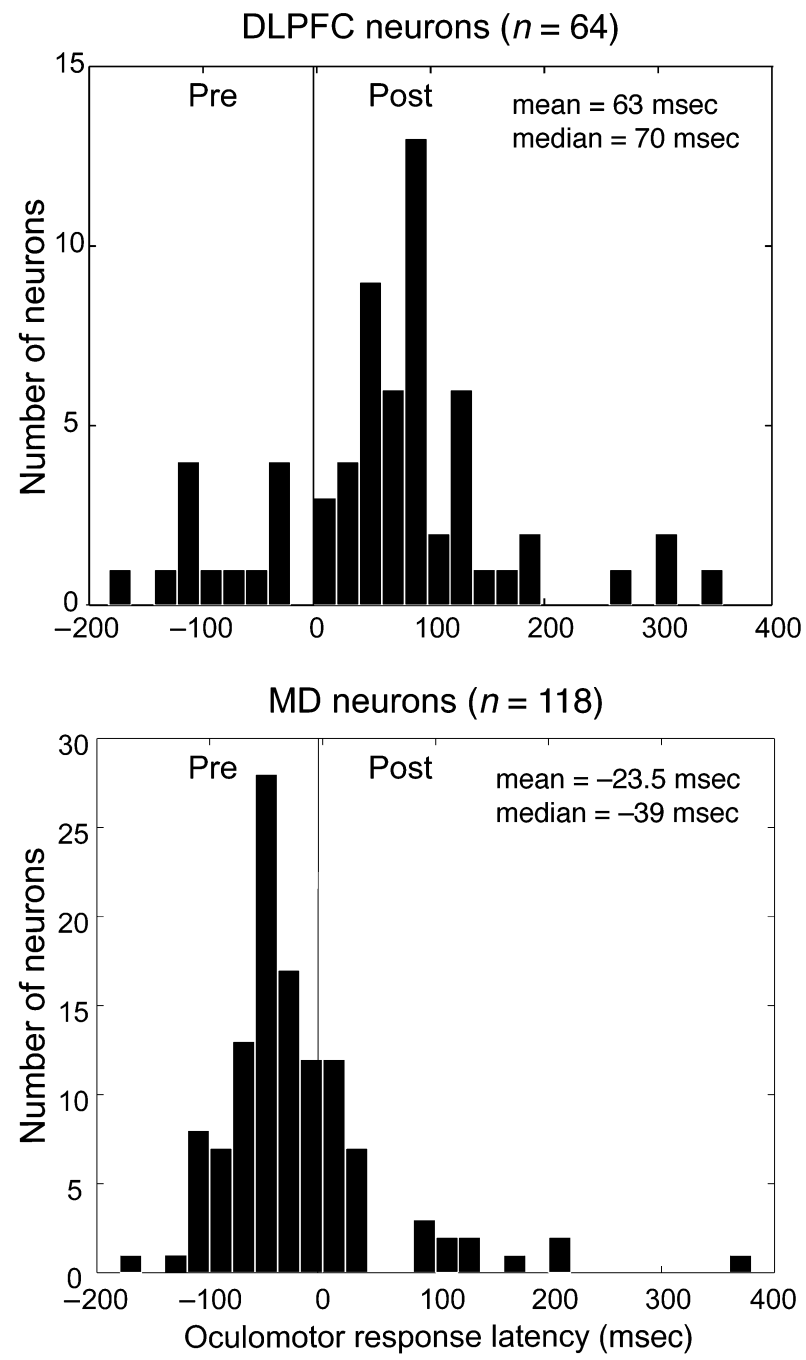

Figure 6. Distribution of latencies for response period activity. The latency for response period activity was defined as the duration from the initiation of the saccadic eye movement to the initiation of response period activity. Note that more postsaccadic neurons were present in the dorsolateral prefrontal cortex (DLPFC), whereas more presaccadic neurons were present in the mediodorsal (MD) nucleus.

lateral to the recording hemisphere. The contralateral bias was not statistically significant $\left(\chi^{2}=1.714, p>.05\right)$.

In the MD (Watanabe \& Funahashi, 2004a), the latencies for response period activity were distributed from -170 to $370 \mathrm{msec}$; the mean was $-23 \mathrm{msec}$, and the median was $-39 \mathrm{msec}$. As can be seen in Figure 6, 74\% of the neurons having response period activity showed presaccadic activity, and $26 \%$ showed only postsaccadic activity. Most (78\%) of the MD neurons having presaccadic activity exhibited directional selectivity (Figure 7). Among these neurons, 59\% had the best directions toward the visual field contralateral to the recording hemisphere, whereas $26 \%$ had the best directions ipsilateral to the recording hemisphere. A significant contralateral bias $\left(\chi^{2}=12.81, p<.01\right)$ was observed in the distribution of the best directions for presaccadic activity. On the other hand, most (74\%) of the MD neurons having postsaccadic activity exhibited omnidirectional activity, and only $26 \%$ exhibited directionally selective activity (Figure 7). No significant contralateral bias was observed in the distribution of the best directions for directionally selective postsaccadic activity $\left(\chi^{2}=0.14, p>.05\right)$.

\section{Comparison of Task-Related Activity Between the DLPFC and the MD}

The percentage of neurons with delay period activity among all the neurons that showed task-related activity and the percentages of neurons with either directional or omnidirectional delay period activity are similar between the DLPFC and the MD. In addition, the temporal patterns of delay period activity that we observed in the MD (tonic sustained excitation, gradual increase, gradual decrease, and inhibition) were the same as those observed in the DLPFC (Chafee \& Goldman-Rakic, 1998; Funahashi et al., 1989; Funahashi \& Takeda, 2002). Thus, we found a great similarity in the characteristics of delay period activity between the DLPFC and the MD. This similarity could be due to strong anatomical connections between these two structures (Goldman-Rakic \& Porrino, 1985; Kievit \& Kuypers, 1977; Rouiller et al., 1999).

However, several differences were also found in the characteristics of delay period activity between the DLPFC and the MD. First, the distributions of the best directions were different between the DLPFC and the MD. In both areas, the best directions of delay period activity were distributed among most of the directions around the FP. A significant contralateral bias of the best directions was
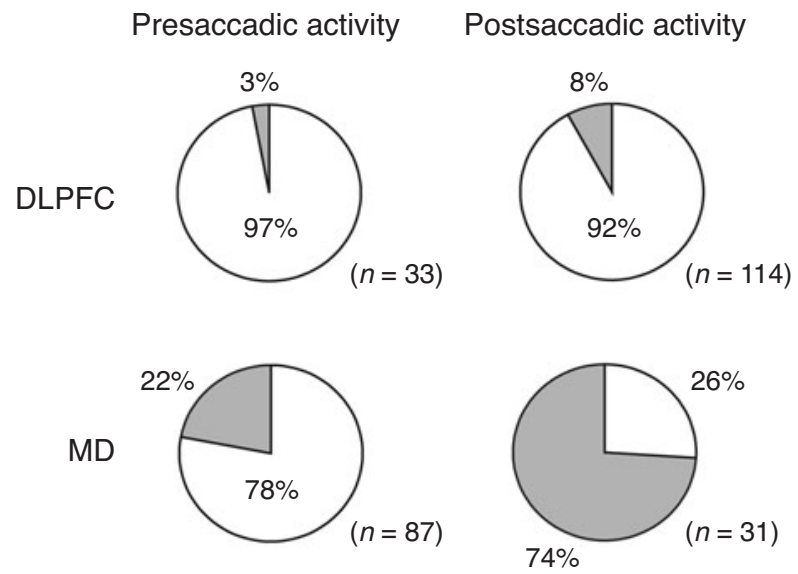

Directional activity Omnidirectional activity

Figure 7. Directional selectivity of pre- and postsaccadic activity observed in dorsolateral prefrontal cortex (DLPFC) and mediodorsal (MD) neurons. The DLPFC data are based on Takeda and Funahashi (2002). The MD data are based on Watanabe and Funahashi (2004a). 
observed in the DLPFC (Funahashi et al., 1989; Takeda \& Funahashi, 2002). In contrast, a significant contralateral bias was not observed in the MD, although most of the best directions were directed toward the contralateral visual field (Watanabe \& Funahashi, 2004a). Second, the mean tuning indices were different between the DLPFC and the MD. The mean tuning index of both excitatory and inhibitory activity was broader in the $\mathrm{MD}\left(59^{\circ}\right.$ for excitatory activity; Watanabe \& Funahashi, 2004a) than in the DLPFC $\left(27^{\circ}\right.$ for excitatory activity; Funahashi et al., 1989). An anatomical study indicated that the MD has connections with the bilateral DLPFC, although the ipsilateral projections between the DLPFC and the parvocellular MD are much stronger than the contralateral projections (Preuss \& Goldman-Rakic, 1987). DLPFC neurons with directional delay period activity represent spatial information of visual cues presented mainly in the contralateral visual field (Funahashi et al., 1989; Takeda \& Funahashi, 2002), and the MD has connections with the bilateral DLPFC. Therefore, single MD neurons might receive directional information from both sides of the DLPFC. This anatomical feature would therefore explain why MD neurons have wider memory fields, as well as why no contralateral bias was observed in MD neurons.

A comparison of the characteristics of cue period activity between the DLPFC and the MD reveals functional similarities between these two structures. Possible sources of visual inputs to the MD would be the DLPFC (GoldmanRakic \& Porrino, 1985), the superior colliculus (Lynch, Hoover, \& Strick, 1994), and the substantia nigra pars reticulata (Ilinsky, Jouandet, \& Goldman-Rakic, 1985). The characteristics of cue period activity are very similar among these structures. Although the DLPFC and the MD have strong reciprocal connections, the characteristics of delay period activity show some difference between the DLPFC and the MD, suggesting that cue period activity observed in the MD may be a product of the combined inputs from the DLPFC, the superior colliculus, and the substantia nigra.

We observed striking differences when we compared the characteristics of response period activity between the DLPFC and the MD. The proportion of neurons with response period activity among neurons with task-related activity was significantly lower in the DLPFC than in the MD. More neurons exhibited presaccadic activity in the MD than in the DLPFC, and more presaccadic neurons in the MD exhibited omnidirectional activity. These results indicate that the participation of the MD in ODR task performance is different from that of the DLPFC, especially with regard to the control and execution of motor responses, and suggest that the MD participates in the control and execution of saccadic eye movements more directly than the DLPFC does. On the other hand, although some DLPFC neurons exhibited presaccadic activity, most saccade-related DLPFC activity was postsaccadic, and hence, this postsaccadic activity is considered to be a feedback signal from oculomotor structures for manipulating task-related activity, especially delay period activity (Funahashi \& Kubota, 1994; Funahashi \& Takeda, 2002; Goldman-Rakic, Funahashi, \& Bruce, 1990). Sommer and Wurtz (2004) indicated that presaccadic activity observed in the MD is a corollary discharge for internal monitoring of saccadic eye movement, which is transmitted from the superior colliculus to the frontal eye field. The presaccadic MD activity observed in the present study may be a corollary discharge from the superior colliculus and may also play a role in controlling or modulating delay period activity in the DLPFC through thalamo-cortical afferent projections. The fact that a rather large population of presaccadic activity $(22 \%)$ exhibited omnidirectional selectivity may also support the latter notion, since omnidirectional presaccadic activity does not seem to participate directly in the execution of eye movements toward any particular direction.

\section{INFORMATION THAT EACH TASK-RELATED ACTIVITY REPRESENTS}

The present results indicate that, although the temporal patterns and the proportions of task-related activity and directionally selective activity are similar between the DLPFC and the MD, the characteristics of task-related activity were not identical. These results suggest that there are some differences in how these two areas participate in spatial working memory processes. To further clarify the functional similarities and differences between the DLPFC and the MD, it is important to identify the information represented by each task-related activity while monkeys perform spatial working memory tasks. Such studies have been performed in the DLPFC (Funahashi et al., 1993; Niki \& Watanabe, 1976; Takeda \& Funahashi, 2002). For example, Takeda and Funahashi (2002) found that cue period activity and most delay period activity represented retrospective (sensory) information, whereas most response period activity represented prospective (motor) information. Therefore, it is important to compare information represented by taskrelated activity between the DLPFC and the MD to understand the functional similarities and differences between these two areas.

\section{The Method for Determining What Information Task-Related Activity Represents}

To determine what information is represented by each task-related activity observed in each DLPFC or MD neuron, we compared the directional characteristics for the same task-related activity between ODR and R-ODR tasks that were observed in individual neurons. We first constructed tuning curves for the same task-related activity under two task conditions and determined the best direction in each task condition $\left(D_{\mathrm{ODR}}\right.$ for the best direction under the ODR task and $D_{\mathrm{R}-\mathrm{ODR}}$ for the best direction under the R-ODR task). In the ODR task, the monkeys were required to make a saccadic eye movement 
toward the location at which the visual cue had been presented, whereas in the R-ODR task, the monkeys were required to make a saccadic eye movement $90^{\circ}$ clockwise from the direction in which the visual cue had been presented. Therefore, if the difference between the best directions in the two tasks $\left(D_{\mathrm{R}-\mathrm{ODR}}-D_{\mathrm{ODR}}\right)$ was less than $45^{\circ}$, we could conclude that this task-related activity represents the location of the visual cue. On the other hand, if this difference $\left(D_{\mathrm{R}-\mathrm{ODR}}-D_{\mathrm{ODR}}\right)$ was more than $45^{\circ}$ and less than $135^{\circ}$, we could conclude that this taskrelated activity represents the direction of the saccade. Using this method, we analyzed in detail 121 DLPFC neurons and $98 \mathrm{MD}$ neurons that exhibited task-related activity in the same task event in both ODR and R-ODR tasks, and determined whether each task-related activity observed in DLPFC and MD neurons represents the location of the visual cue or the direction of the saccade
(Takeda \& Funahashi, 2002; Watanabe \& Funahashi, 2004b).

\section{What Information Does Cue Period Activity Represent?}

Using 41 DLPFC and 19 MD neurons exhibiting directional cue period activity in both tasks, we compared $D_{\text {R-ODR }}$ with $D_{\text {ODR. }}$ A figure at the top-left position of Figure 8 shows an example of the tuning curve for directional cue period activity. The best directions were almost identical in the two tasks. Figures on the top row of Figure 8 show the distribution of the differences of the best directions between the two tasks $\left(D_{\mathrm{R}-\mathrm{ODR}}-D_{\mathrm{ODR}}\right)$ for all DLPFC and MD neurons that exhibited directional cue period activity. These differences for the best directions were distributed between $-45^{\circ}$ and $45^{\circ}$ for both DLPFC and MD neurons. Therefore, these results
A Cue period activity
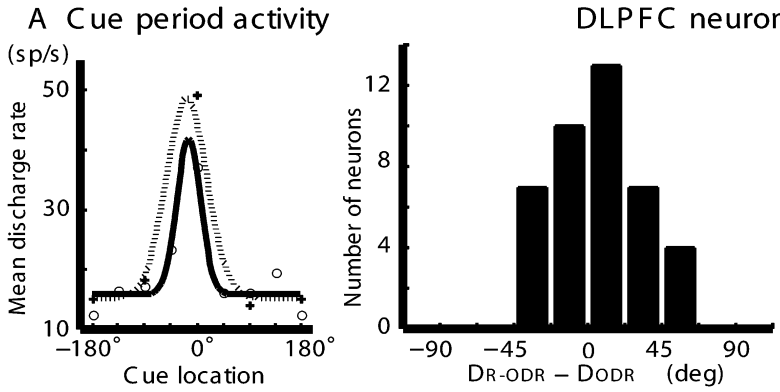

B Delay period activity
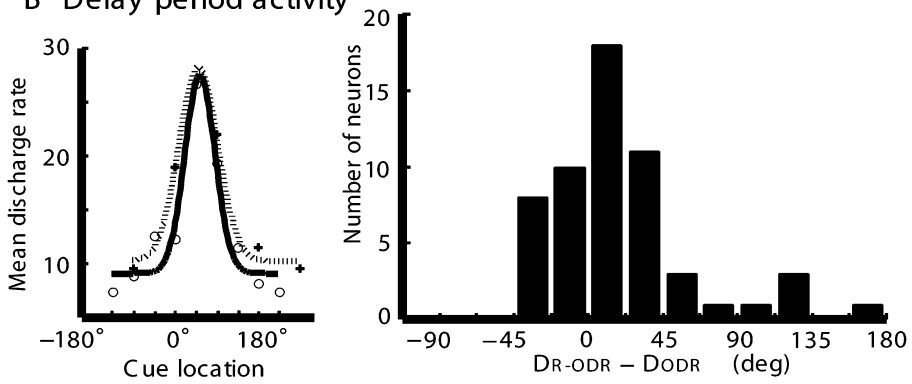

C Response period activity
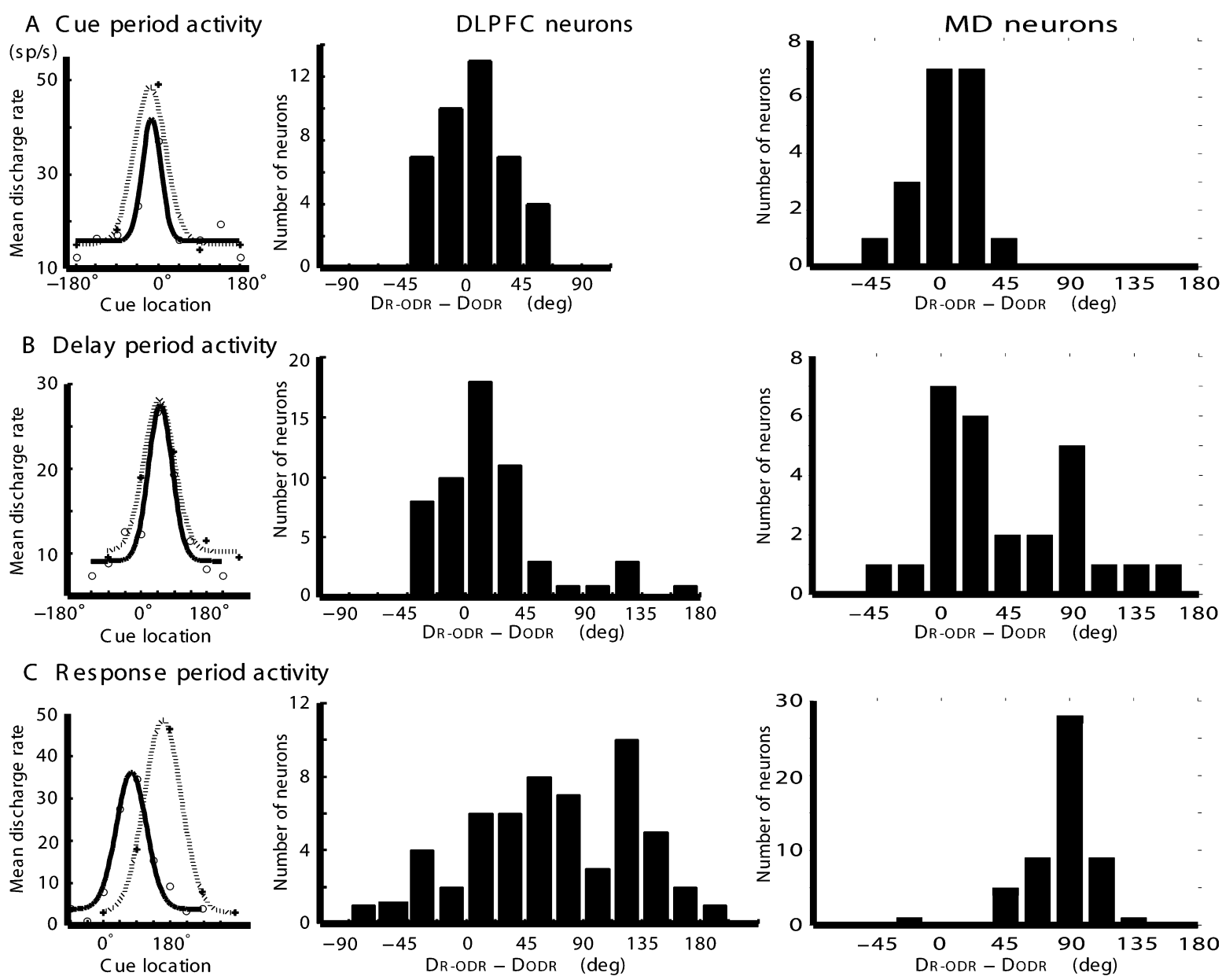

- ODR task

i' R-ODR task

Figure 8. Left: Examples of tuning curves for the same task-related activity recorded from the same dorsolateral prefrontal cortex (DLPFC) neurons under oculomotor delayed response (ODR) task and rotatory ODR (R-ODR) task conditions. Middle: The distribution of the difference of the best directions $\left(D_{\mathrm{R}-\mathrm{ODR}}-D_{\mathrm{ODR}}\right)$ for three task-related activities observed in the DLPFC. Right: The distribution of the difference of the best directions $\left(D_{\mathrm{R}-\mathrm{ODR}}-D_{\mathrm{ODR}}\right)$ for three task-related activities observed in mediodorsal (MD) neurons. 
indicate that directional cue period activity represents the location of the visual cue for all the recorded DLPFC and MD neurons that exhibited this activity (Figure 9). Since dense reciprocal connections are present between the DLPFC and the MD (Giguere \& Goldman-Rakic, 1988; Goldman-Rakic \& Porrino, 1985; Kievit \& Kuypers, 1977), the similarity in the characteristics of cue period activity between the DLPFC and the MD indicates strong functional interactions between these two structures through these anatomical connections.

\section{What Information Does Delay Period Activity Represent?}

Fifty-six DLPFC and 27 MD neurons exhibited delay period activity in both tasks. By comparing the best directions for delay period activity between the two tasks, we found that directional delay period activity can be classified into two groups: one that represents the location of the visual cue and another that represents the direction of the saccade. A figure at the middle-left position of Figure 8 shows an example of the tuning curve for delay period activity under two task conditions. The best directions were almost identical in the two tasks. Therefore, we could conclude that this neuron's delay period activity represents the location of the visual cue. Figures in the middle row of Figure 8 show the distribution of the differences for best directions between the two tasks. The differences for the best directions $\left(D_{\mathrm{R}-\mathrm{ODR}}-\right.$ $\left.D_{\text {ODR }}\right)$ were distributed between $-45^{\circ}$ and $160^{\circ}$ for DLPFC neurons and between $-45^{\circ}$ and $135^{\circ}$ for MD neurons. In $86 \%$ of the DLPFC neurons and $56 \%$ of the MD neurons, the differences between the best directions were between $-45^{\circ}$ and $45^{\circ}$, indicating that these neu- rons' delay period activity represents the location of the visual cue (Figure 9). However, in $13 \%$ of the DLPFC neurons and $41 \%$ of the MD neurons, the differences for the best directions were greater than $45^{\circ}$, indicating that delay period activity represents the direction of the saccade (Figure 9). These results indicate that both DLPFC and MD neurons can retain either retrospective or prospective information during the delay period as directional delay period activity. In DLPFC neurons, the great majority of delay period activity represented the location of the visual cue. However, in MD neurons, about half of delay period activity represented the location of the visual cue, whereas the remaining half of such activity represented the direction of the saccade.

The observation that most delay period activity in the DLPFC during spatial delayed response performance represents the location of the visual cue had also been noted previously by Niki and Watanabe (1976) and Funahashi et al. (1993). For example, Funahashi et al. (1993) examined the same neuron's delay period activity in a delayed prosaccade task and a delayed antisaccade task and found that $70 \%$ of directional delay period activity represented the location of the visual cue, whereas 30\% represented the direction of the saccade. In addition, in tasks that require nonspatial information processing, such as a delayed matching-to-sample task and a delayed paired associate task, more delay period activity in the DLPFC has been shown to represent retrospective information, such as the sample stimuli, although delay period activity can represent either retrospective or prospective information (Quintana \& Fuster, 1999; Rainer \& Miller, 2002; Rainer, Rao, \& Miller, 1999). These results indicate that, in general, more DLPFC neurons retain retrospective informa-
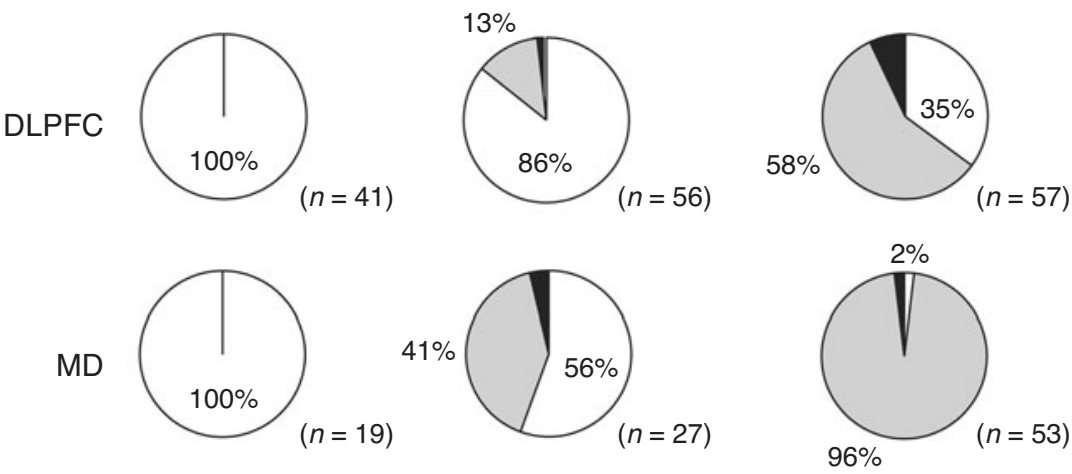

Activity encoding the location of the visual cue

Activity encoding the direction of the saccade

Unclassified

Figure 9. The proportion of task-related activity representing either information regarding the location of the visual cue or information regarding the direction of the saccade in dorsolateral prefrontal cortex (DLPFC) and mediodorsal (MD) neurons. 
tion, such as sample or cue stimuli, than retain prospective information, such as motor response directions.

As is shown in Figure 9, the results obtained in the DLPFC differ from those obtained in the MD, in that the proportion of delay period activity in the MD that represented retrospective information was similar to the proportion of delay period activity that represented prospective information. These results indicate that although both DLPFC and MD neurons participate in either retrospective or prospective information processing, a greater percentage of MD neurons participate in prospective information processing. Therefore, although the DLPFC and the MD have strong reciprocal connections (Giguere \& Goldman-Rakic, 1988; Goldman-Rakic \& Porrino, 1985 ; Kievit \& Kuypers, 1977), these results suggest that information processing that occurs in the MD is not the same as that in the DLPFC.

\section{What Information Does Response Period Activity Represent?}

For response period activity, the best directions for 57 DLPFC neurons and $53 \mathrm{MD}$ neurons were compared between the two tasks. A panel at the bottom-left position of Figure 8 shows an example of the tuning curves of response period activity under two task conditions. The difference between the best directions was nearly $90^{\circ}$. Therefore, the result indicates that this response period activity represents the direction of the saccade. The panels in the bottom row of Figure 8 show the distribution of the differences of the best directions for directional response period activity between the two tasks.

In DLPFC neurons, 58\% showed response period activity representing the direction of the saccade, whereas $35 \%$ showed response period activity representing the location of the visual cue (Figure 9). Most of the DLPFC neurons that had response period activity representing the location of the visual cue showed postsaccadic activity and also exhibited delay period activity representing the location of the visual cue. In contrast, in the MD neurons, the differences for the best directions $\left(D_{\mathrm{R}-\mathrm{ODR}}-\right.$ $\left.D_{\text {ODR }}\right)$ were distributed between $45^{\circ}$ and $135^{\circ}$, indicating that almost all response period activity $(96 \%)$ represented the direction of the saccade (Figure 9).

As is shown in Figure 9, the results obtained from the MD are in sharp contrast to those obtained in the DLPFC. Takeda and Funahashi (2002) showed that a large proportion of postsaccadic activity represented the location of the visual cue, whereas presaccadic activity often represented the direction of the saccade. They also showed that, in the DLPFC, many postsaccadic activities and even presaccadic activities represented the location of the visual cue. Therefore, it is suggested that both preand postsaccadic activity functions not only as a signal to control saccadic eye movements, but also as a signal to modulate task-related activities in the DLPFC. In contrast, most MD neurons with directional response period activity showed presaccadic activity, and such presac- cadic activity represented information regarding the direction of the saccade. Therefore, presaccadic activity observed in MD neurons apparently functions as a signal to control saccadic eye movements. Thus, although both the DLPFC and the MD participate in cognitive processes, such as working memory processes, the role of the MD in these processes is different from that of the DLPFC.

\section{CONCLUSION}

To examine the MD's participation in working memory, we analyzed the characteristics of task-related activities while monkeys performed spatial working memory tasks and compared these characteristics between the DLPFC and the MD. Same task-related activities were observed in both MD neurons and DLPFC neurons. Temporal and directional preferences for these task-related activities were basically similar between these two areas. However, response period activity was more frequently observed in the MD than in the DLPFC. Presaccadic activity was predominant in the MD, whereas postsaccadic activity was predominant in the DLPFC. Most of the task-related activity exhibited directional selectivity in both areas. The ratios of neurons that exhibited directional cue or delay period activity was similar in both areas, whereas more neurons showed omnidirectional response period activity in the MD than in the DLPFC. Difference in the bias for directional selectivity for delay period activity was observed between these two areas. In addition, although task-related activities recorded from both areas represent either retrospective (e.g., sensory) or prospective (e.g., motor) information, more DLPFC neurons represent retrospective information, whereas more MD neurons represent prospective information. These results indicate that although the MD participates in the spatial working memory process, the way it participates in this process is different between these two areas, in that the MD participates more in motor control aspects than the DLPFC does.

\section{REFERENCES}

Alexander, G. E., DeLong, M. R., \& Strick, P. L. (1986). Parallel organization of functionally segregated circuits linking basal ganglia and cortex. Annual Review of Neuroscience, 9, 357-381.

BAdDEley, A. (1986). Working memory. Oxford: Oxford University Press.

Chafee, M. V., \& Goldman-Rakic, P. S. (1998). Matching patterns of activity in primate prefrontal area $8 \mathrm{a}$ and parietal area 7 ip neurons during a spatial working memory task. Journal of Neurophysiology, 79, 2919-2940.

de Zubicaray, G. I., McMahon, K., Wilson, S. J., \& Muthiah, S. (2001). Brain activity during the encoding, retention, and retrieval of stimulus representations. Learning \& Memory, 8, 243-251.

ElliotT, R., \& Dolan, R. J. (1999). Differential neural responses during performance of matching and nonmatching to sample tasks at two delay intervals. Journal of Neuroscience, 19, 5066-5073.

Funahashi, S., BRUCE, C. J., \& GoldMan-RAKIC, P. S. (1989). Mnemonic coding of visual space in the monkey's dorsolateral prefrontal cortex. Journal of Neurophysiology, 61, 331-349. 
Funahashi, S., Bruce, C. J., \& Goldman-Rakic, P. S. (1990). Visuospatial coding in primate prefrontal neurons revealed by oculomotor paradigms. Journal of Neurophysiology, 63, 814-831.

Funahashi, S., BRUCE, C. J., \& Goldman-R AKIC, P. S. (1991). Neuronal activity related to saccadic eye movements in the monkey's dorsolateral prefrontal cortex. Journal of Neurophysiology, 65, 14641483.

Funahashi, S., Chafee, M. V., \& Goldman-Rakic, P. S. (1993). Prefrontal neuronal activity in rhesus monkeys performing a delayed anti-saccade task. Nature, 365, 753-756.

Funahashi, S., Inoue, M., \& Kubota, K. (1997). Delay-period activity in the primate prefrontal cortex encoding multiple spatial positions and their order of presentation. Behavioural Brain Research, 84, 203-223.

FUNAHASHI, S., \& KubOTA, K. (1994). Working memory and prefrontal cortex. Neuroscience Research, 21, 1-11.

FUNAHASHI, S., \& TAKEDA, K. (2002). Information processes in the primate prefrontal cortex in relation to working memory processes. $R e$ views in the Neurosciences, 13, 313-345.

FUSTER, J. M. (1973). Unit activity in prefrontal cortex during delayedresponse performance: Neuronal correlates of transient memory. Journal of Neurophysiology, 36, 61-78.

FUSTER, J. M. (1990). Inferotemporal units in selective visual attention and short-term memory. Journal of Neurophysiology, 64, 681-697.

Fuster, J. M. (1997). The prefrontal cortex. Philadelphia: LippincottRaven.

Fuster, J. M., \& AleXANDER, G. E. (1971). Neuron activity related to short-term memory. Science, 173, 652-654.

Fuster, J. M., \& AleXANDER, G. E. (1973). Firing changes in cells of the nucleus medialis dorsalis associated with delayed response behavior. Brain Research, 61, 79-91.

Giguere, M., \& GoldMaN-RAKIC, P. S. (1988). Mediodorsal nucleus: Areal, laminar, and tangential distribution of afferents and efferents in the frontal lobe of rhesus monkeys. Journal of Comparative Neurology, 277, 195-213.

GNADT, J. W., \& ANDERSEN, R. A. (1988). Memory related motor planning activity in posterior parietal cortex of macaque. Experimental Brain Research, 70, 216-220.

GolDMAN-RAKIC, P. S. (1987). Circuitry of primate prefrontal cortex and regulation of behavior by representational memory. In J. M. Brookhart \& V. B. Mountcastle (Series Eds.) \& F. Plum (Vol. Ed.), Handbook of physiology: Sect. 1. The nervous system. Vol. V. Higher functions of the brain (pp. 373-417). Bethesda, MD: American Physiological Society.

GOLDMAN-RAKIC, P. S. (1996a). The prefrontal landscape: Implications of functional architecture for understanding human mentation and the central executive. Philosophical Transactions of the Royal Society of London: Series B, 351, 1445-1453.

GoLDMAN-RAKIC, P. S. (1996b). Regional and cellular fractionation of working memory. Proceedings of the National Academy of Sciences, 93, 13473-13480.

Goldman-Rakic, P. S., Funahashi, S., \& Bruce, C. J. (1990). Neocortical memory circuits. Cold Spring Harbor Symposium on Quantitative Biology, 55, 1025-1038.

Goldman-RAKIC, P. S., \& Porrino, L. J. (1985). The primate mediodorsal (MD) nucleus and its projection to the frontal lobe. Journal of Comparative Neurology, 242, 535-560.

HiKosaKa, O., SaKamoto, M., \& UsUi, S. (1989). Functional properties of monkey caudate neurons: I. Activities related to saccadic eye movements. Journal of Neurophysiology, 61, 780-798.

ILINSKY, I. A., JOUANDET, M. L., \& GoldMAN-RAKIC, P. S. (1985). Organization of the nigrothalamocortical system in the rhesus monkey. Journal of Comparative Neurology, 236, 315-330.

IsserofF, A., Rosvold, H. E., GALKIN, T. W., \& GoldMan-RAKIC, P. S. (1982). Spatial memory impairments following damage to the mediodorsal nucleus of the thalamus in rhesus monkeys. Brain Research, 232, 97-113.

KIEVIT, J., \& KUYPERS, H. G. (1977). Organization of the thalamo-cortical connexions to the frontal lobe in the rhesus monkey. Experimental Brain Research, 29, 299-322.
LYNCH, J. C., Hoover, J. E., \& STRICK, P. L. (1994). Input to the primate frontal eye field from the substantia nigra, superior colliculus, and dentate nucleus demonstrated by transneuronal transport. Experimental Brain Research, 100, 181-186.

MiLLER, E. K., LIN, L., \& DESIMONE, R. (1993). Activity of neurons in anterior inferior temporal cortex during a short-term memory task. Journal of Neuroscience, 13, 1460-1478.

MiYASHitA, Y., \& CHANG, H. S. (1988). Neuronal correlate of pictorial short-term memory in the primate temporal cortex. Nature, 331, 6870 .

Monchi, O., Petrides, M., Petre, V., Worsley, K., \& Dagher, A. (2001). Wisconsin Card Sorting revisited: Distinct neural circuits participating in different stages of the task identified by event-related functional magnetic resonance imaging. Journal of Neuroscience, 21, 7733-7741

NIKI, H. (1974). Differential activity of prefrontal units during right and left delayed response trials. Brain Research, 70, 346-349.

Niki, H., \& Watanabe, M. (1976). Prefrontal unit activity and delayed response: Relation to cue location versus direction of response. $\underline{\text { Brain }}$ Research, 105, 79-88.

PARKER, A., EACOTT, M. J., \& GAFFAN, D. (1997). The recognition memory deficit caused by mediodorsal thalamic lesion in non-human primates: A comparison with rhinal cortex lesion. European Journal of Neuroscience, 9, 2423-2431.

PrEUSS, T. M., \& GoLDMAN-RAKIC, P. S. (1987). Crossed corticothalamic and thalamocortical connections of macaque prefrontal cortex. Journal of Comparative Neurology, 257, 269-281.

QuintANA, J., \& Fuster, J. M. (1999). From perception to action: Temporal integrative functions of prefrontal and parietal neurons. Cerebral Cortex, 9, 213-221.

Rainer, G., AsaAd, W. F., \& Miller, E. K. (1998). Memory fields of neurons in the primate prefrontal cortex. Proceedings of the National Academy of Sciences, 95, 15008-15013.

RAINER, G., \& MILlER, E. K. (2002). Time course of object-related neural activity in the primate prefrontal cortex during a short-term memory task. European Journal of Neuroscience, 15, 1244-1254.

Rainer, G., Rao, S. C., \& Miller, E. K. (1999). Prospective coding for objects in primate prefrontal cortex. Journal of Neuroscience, $\mathbf{1 9}$, 5493-5505.

RAO, S. C., Rainer, G., \& Miller, E. K. (1997). Integration of what and where in the primate prefrontal cortex. Science, 276, 821-824.

Rouiller, E. M., Tanne, J., Moret, V., \& BoussaOud, D. (1999). Origin of thalamic inputs to the primary, premotor, and supplementary motor cortical areas and to area 46 in macaque monkeys: A multiple retrograde tracing study. Journal of Comparative Neurology, 409. 131-152.

Sommer, M. A., \& Wurtz, R. H. (2004). What the brain stem tells the frontal cortex: I. Oculomotor signals sent from superior colliculus to frontal eye field via mediodorsal thalamus. Journal of Neurophysiology, 91, 1381-1402.

TAKEDA, K., \& FunAHASHI, S. (2002). Prefrontal task-related activity representing visual cue location or saccade direction in spatial working memory tasks. Journal of Neurophysiology, 87, 567-588.

TANiBuChI, I., \& GolDMAN-RAKIC, P. S. (2003). Dissociation of spatial-, object-, and sound-coding neurons in the mediodorsal nucleus of the primate thalamus. Journal of Neurophysiology, 89, 1067-1077.

Watanabe, Y., \& FunAHASHI, S. (2004a). Neuronal activity throughout the primate mediodorsal nucleus of the thalamus during oculomotor delayed response: I. Cue-, delay-, and response-period activity. Journal of Neurophysiology, 92, 1738-1755.

Watanabe, Y., \& Funahashi, S. (2004b). Neuronal activity throughout the primate mediodorsal nucleus of the thalamus during oculomotor delayed response: II. Activity encoding visual versus motor signal. Journal of Neurophysiology, 92, 1756-1769.

WiLSON, F. A. W., O SCALAIDHE, S. P., \& GolDMAN-RAKIC, P. S. (1993). Dissociation of object and spatial processing domains in primate prefrontal cortex. Science, 260, 1955-1958.

(Manuscript received August 3, 2004; revision accepted for publication October 11, 2004.) 